\title{
The Effect of Decision-Criteria Dynamics on Network Selection for Group Calls in Heterogeneous Wireless Networks
}

\author{
Enoruwa Obayiuwana \\ Department of Electronic and Electrical Engineering \\ Obafemi Awolowo University, \\ Ile-Ife, Nigeria
}

\author{
Joseph Orimolade \\ Department of Electrical and Electronics Engineering \\ Federal University of Agriculture, \\ Abeokuta, Nigeria
}

\begin{abstract}
This paper focuses on the network selection for group calls in heterogeneous wireless networks (HWNs) with dynamic criteria. A group call comprises multiple independent calls, such as: voice, file-download, video-streaming, online-gaming sessions, etc. that are activated concurrently on a multimode mobile node (MN). The next generation wireless networks (NGWNs) have the capability to support group calls from multimode MNs. Network selection for group calls in HWNs is a complex and multi-criteria group decision (MCGDM) problem. Existing works in the literature that addresses the network-selection problem of the vertical handover (VHO) of group calls assumes that the multiple criteria used for making network selection decisions are static. However, in NGWNs, some criteria, such as: MN's speed, network's service price, and network traffic load, are used for making network-selection decisions for VHO that are dynamic. The dynamics of the network selection criteria for VHO of group calls affect the choice of the most suitable selected network. Thus, it is important to consider decision criteria dynamics when making network selection decisions for the VHO of group calls in NGWNs. In this paper, a new network selection algorithm that uses a MULTlplicative-form with Multi-Objective Optimization Ratio Analysis (MULTIMOORA) for making network selection decisions for the VHO of group calls is proposed. An investigation into the effect of decision criteria dynamics on VHO decisions for group calls is carried out. The performance of the MULTIMOORA is validated by comparing it with TOPSIS, a well- known MCGDM algorithm. The performance of TOPSIS is seen to be unstable at high MN speed regions, unlike MULTIMOORA. The simulation results show the effectiveness of the proposed scheme for making network- selection decisions for group calls in HWNs with dynamic multi-criteria.
\end{abstract}

\section{Keywords}

Dynamic multi-criteria, Group calls, Network selection, Multicriteria group decision- making

\section{INTRODUCTION}

The current decade has witnessed an unprecedented growth in wireless communication- network's capabilities [1, 2]. The demands for ubiquitous network coverage and services are constantly on the rise. A plethora of network services, such as: voice, file-download, video-streaming and online gaming are now offered worldwide by most wireless network operators [3]. These network services present diverse range of quality of service (QoS) requirements. Next-generation multimode mobile nodes (MNs) have the capability to simultaneously support two or more different classes of calls [4]. Group calls comprise multiple independent calls, such as: voice, file-download, video-streaming, online-gaming sessions, which can be activated and sustained simultaneously, while the multimode $\mathrm{MN}$ is attached to an access network within the HWNs. Group calls can consist of dual calls, triple calls, quadruple calls, e.t.c. Fig 1 shows the various multimode MNs making different classes of calls in HWNs. The HWNs integrates the Wireless Local Area Network (WLAN), Universal Mobile Telecommunication Systems (UMTS) and Long-Term Evolution (LTE) networks together [5]. WLAN has the lowest support for mobility, and the least coverage area [6]. LTE supports the highest mobility among the three networks [6, 7]. WLAN can support $25-30 \mathrm{~km} / \mathrm{h}$; UMTS can support $90-100 \mathrm{~km} / \mathrm{h}$ and LTEs can support up to $150 \mathrm{~km} / \mathrm{h}[8]$. Each of these networks would support differently, the QoS requirement of a given group call from the MN. In Fig. 1, MN1 is having a voice (single) call; MN2 is having voice and file-download (dual) calls; and MN3 is having voice, file-download, and videostreaming (triple) calls. The MNs are moving at different speed within the HWNs.

Assume that an MN at a given time has initiated a video-streaming from an access point/base station (AP/BS) on an access network; and while the initiated video-streaming is ongoing, assume that the $\mathrm{MN}$ receives an incoming voice call from the same AP/BS. The access network should be able to support both calls simultaneously without dropping/terminating the existing video-streaming call, or blocking the in-coming voice call. On the receiver's end, the MN should not perceive any degradation in QoS of the existing/new call throughout the duration of the call sessions. Achieving these goals for group calls are serious challenges in HWNs. A wireless network in HWNs may not be able to provide the desired QoS for the group calls, due to the initiation of new call(s) concurrently with the existing call(s). It is imperative for the HWNs to be able to execute a fast and seamless vertical handover (VHO) of the group calls to different wireless access network within the HWNs that can satisfy the newly required QoS for the group calls. 


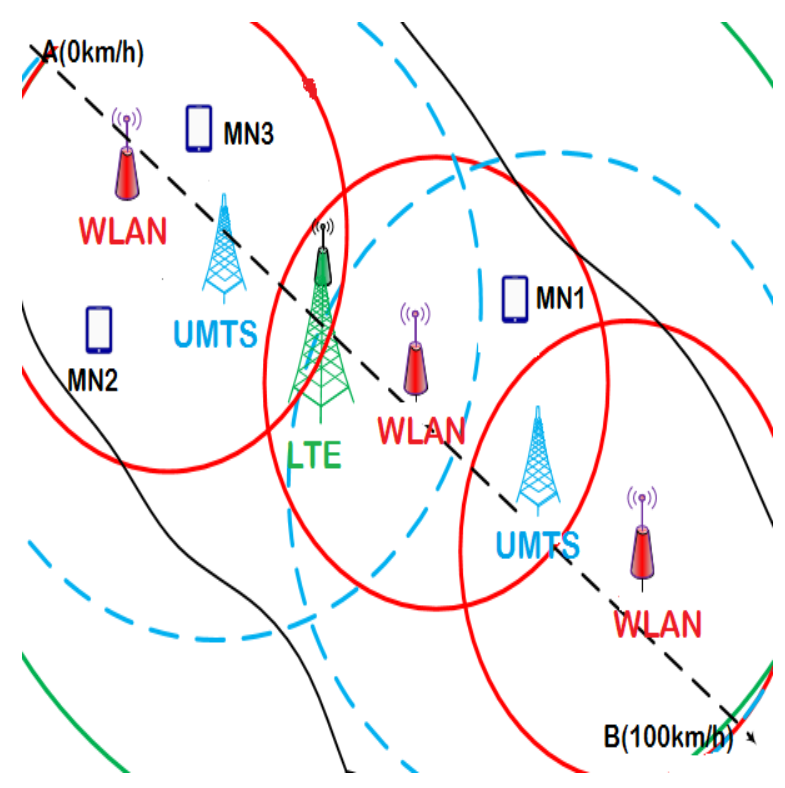

Fig. 1. Communicating multimode mobile nodes in HWNs

Switching from one optimum access network technology to another allows an MN to achieve always best connection (ABC) within HWNs [9-12]. This also ensures that the MNs exploit the full benefits of the network's profile configurations offered by the diverse wireless networks. The VHO process allows such benefits to be realized by allowing effective, fast and seamless switching between the different wireless networks with minimal VHO latency. Network selection in HWNs is determined by multiple- wireless network criteria. Network selection in HWNs is a multi-criteria decision problem. Network selection for group calls is a multi-criteria group decision problem.

Access network selection in HWNs is intrinsically a manifold problem; and it requires the consideration of numerous parameters for efficient VHO decision- making. Classical network selection schemes are based on single-criterion parameters, such as Received Signal Strength (RSS) or Signal-to- Noise Ratio (SNR). Single criterion schemes are inefficient for network selection in HWNs, because of the effects of the heterogeneity of network parameter standards across the HWNs.

The VHO decision- process in HWNs involves complex and often conflicting multi-criteria. The VHO decision- process in HWNs can be modelled as multi-criteria decision- making (MCDM) problems. VHO decisions for group calls can be modelled as multi-criteria group-decision (MCGDM) problems. The MCGDM is a logical extension of MCDMs for group- decision- making problems. MCDM is an advanced tool of Optimization research technique for resolving multiple and often conflicting criteria decision problems. A lot of MCDM schemes, such as: Simple Additive Weighting (SAW), Multiplicative Exponent Weighting (MEW), Grey Relational Analysis (GRA), Analytical Hierarchy Process (AHP), Elimination Et Choix Traduisant la Realite (ELECTRE), Technique for Order Preference by Similarity to Ideal Solutions (TOPSIS), and VlseKriterijumska Optimizacija I Kompromisno (VIKOR) have been utilized in HWNs, [9, 13,-19].

Recently, there has been a growing research interest in developing new and efficient MCDM algorithms. In this paper, a new network selection scheme that uses MULTlplicative-form with MultiObjective Optimization Ratio Analysis (MULTIMOORA) for mak- ing network selection decisions for VHO group calls in HWNs is proposed. The following are the main contributions of this paper:

-A comprehensive review of current network- selection algorithms for single and group calls in HWNs.

- Consideration of decision criteria dynamics and the evaluation of the effects of decision criteria dynamics on network-selection decision- making for group calls in HWNs.

-Application of MULTIMOORA for making VHO decisions for group calls in HWNs.

To the best of our knowledge, this is the first research paper to apply MULTIMOORA to address the problem of network selection for group calls in HWNs. Moreover, it is the first paper to investigate the effect of decision criteria dynamics on VHO network-selection for group calls.

This work provides a new dimension and a realistic approach to wireless- network- selection problems for group calls; and it considers the influence of the priority of classes of calls on wireless network selection for group calls. In order to demonstrate the significance of MULTIMOORA as an effective network handover decision-making mechanism, the simulation results of various case studies are presented.

The rest of this paper is organized as follows: in Section 2 a review of related work is presented; Section 3 discusses the VHOmanagement process; Section 4 introduces the MCDM group- decision problem. Section 5 introduces the MULTIMOORA technique; Section 6 presents MULTIMOORA's application to VHO for group calls using various case studies; while, Section 7 presents the numerical simulation results and subsequent discussion. Finally, Section 8 concludes the paper. A summary of the list abbreviations used in this paper is shown in Table 1

\section{RELATED WORK}

A review of the related work is provided in this section. In [13], semi- and- fully distributed network- selection schemes based on SAW being investigated for HWNs. In [14], the performance of the VIKOR scheme for VHO decisions are compared with SAW and TOPSIS. TOPSIS is observed to have similar performance with VIKOR. However, the effect of criteria weights on the decisions of MCDM schemes are not even considered in the investigations.

The application of ELECTRE in making network selection in HWNs environment has been studied in [15] using three network services, namely: Voice over Internet Protocol (VoIP), videostreaming, and web- browsing. Ourania, et al. in [16] have studied the problem of network selection between General Packet Radio Service (GPRS) and WLAN. A network selection scheme that integrated AHP and GRA is proposed. The weights of the network QoS are assigned, using AHP; while the GPRS and WLAN are ranked, using GRA. In [17], a comparative study of the performance of SAW, MEW, ELECTRE, TOPSIS, GRA and VIKOR for the execution of VHO decisions for voice and data connection applications in HWNs is presented.

In HWNs, the network selection for a single call is influenced by the type of calls requested i.e, voice, video-streaming, filedownload etc. Thus, for group calls the network selection decision would be influenced by the interactive combination of the degree of importance (or the priority) of these independent calls of the group calls.

The problem of Radio Access Technology (RAT) selection for group calls in HWNs has been addressed in [4]. A modified TOPSIS group-decision-making technique that selects the most suitable 
Table 1.

\begin{tabular}{ll} 
& \multicolumn{1}{c}{ List of abbrevaitions } \\
\hline Item & Meaning \\
\hline ABC & Always Best Connection \\
AHP & Analytical Hierarchy Process \\
AP/BS & Access Point/Base Station \\
ELECTRE & Elimination Et Choix Traduisant la Realite \\
GDM & Group Decision Making \\
GPRS & General Packet Radio Service \\
GRA & Grey Relational Analysis \\
HWNs & Heterogeneous Wireless Networks \\
km/h & Kilometer per hour \\
LTE & Long-Term Evolution \\
MbpS & Mega bits per second \\
MCDM & Multi-Criteria Decision-Making \\
MCGDM & Multi-Criteria Group Decision \\
MEW & Multiplicative Exponent Weighting \\
MN & Mobile Node \\
mS & Milli-second \\
MULTIMOORA & Multi-Objective Optimization Ratio Analysis \\
NGWNs & Next Generation Wireless Networks \\
PV & Price Variation \\
QoE & Quality of Experience \\
RAT & Radio Access Technology \\
RSS & Received Signal Strength \\
SAW & Simple Additive Weighting \\
SINR & Signal-to- Noise Ratio \\
TFN & Triangle Fuzzy Numbers \\
TOPSIS & Technique for Order Preference by Similarity \\
& to Ideal Solutions \\
UMTS & Universal Mobile Telecommunication Systems \\
VHO & Vertical Hand Over \\
VIKOR & VlseKriterijumska Optimizacija I Kompromisno \\
WLAN & Wireless Local Area Network \\
\hline &
\end{tabular}

RAT, for a group of different classes of calls from a multimode MN in HWNs, is proposed.

The authors in [20] investigated the effect of the call dynamics of group calls on RAT selection in terms of the probability of having the necessary vertical handoffs in HWNs that support voice, videostreaming and file- download calls, using the Markov chain model. In [21] the author extended the work in [4], by introducing a RAT preference margin, which is a measure of the degree to which the newly preferred RAT is better than the current RAT in reducing the frequency of VHOs for group calls.

From the reviewed related works, the following shortcomings are noted:

-Existing works are limited to the problem of VHO for single calls; and hence, they failed to address the VHO for group calls, except for work done in [4, 20] and [21].

-Existing works have not taken into consideration the dynamic nature of some network-decision criteria, such as: MN's speed, network service price, and network traffic load in their problem formulations.

To address these shortcomings stated above, in this work, a multicriteria network-selection scheme for making VHO decisions for group calls in HWNs with dynamic multi-criteria is proposed. The proposed scheme uses a MULTIMOORA algorithm to make the network selection decisions for the VHO of group calls. To the best of our knowledge, this is the first paper considering the use of a MULTIMOORA algorithm for making network-selection decisions for group calls in HWNs. It is the first paper to investigate the impact of decision criteria dynamics on network-selection decisions for group calls. The following section introduces the VHO management process in NGWNs.

\section{VERTICAL HANDOVEER MANAGEENT PROCESS IN NEXT GENERATION WIRELESS NETWORKS (NGWNS)}

Having an efficient VHO management process is vital for the seamless integration of diverse radio-access networks in NGWNs [2225]. Fig. 2 shows the three integral units of the handover management process.

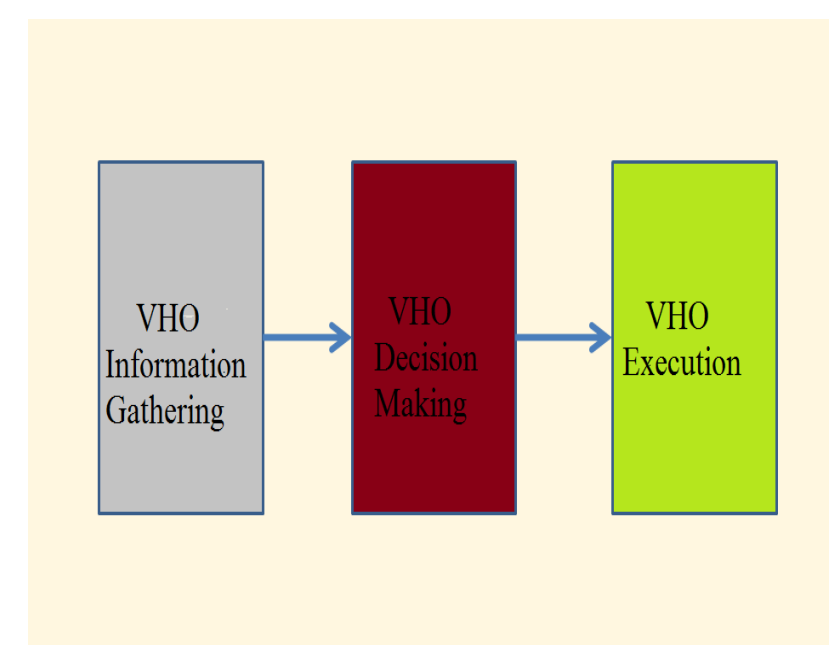

Fig. 2. Handover management process

\subsection{Handover Decision-Information Gathering}

This unit serves as repository and network discovery system for newly available networks [26]. It gathers, manages and evaluates the changes in the gathered handover information. It makes decisions on whether to initiate a VHO process, or not to do so. QoS application information (e.g., required bandwidth and minimum delay), device terminal information (e.g., battery power level and MN speed), network-context information (e.g., network security and network traffic load), and users' context information (e.g., users' locations and preferences) are stored and managed on this unit. When a VHO event is triggered, the vital information needed for a VHO decision is consequently forwarded to the handover decision unit. A handover event is usually triggered when the evaluation of some of the collected key parameters indicates a necessity for handover.

\subsection{Handover Decision-Making}

This is the core of the VHO management process [27]. It is also referred to as the network selector. This unit helps to decide whether the MN should remain connected to its existing network, or switch over to a more suitable network. In the case of switching the MN over to another network, this unit decides the most suitable network for the MN from the set of available network alternatives, using a MCDM algorithm. It also takes into account the criteria necessary 
for VHO decision-making. The decision output of the handover decision unit is passed on to the VHO execution unit.

\subsection{Handover Execution}

The handover execution unit ensures a smooth session transition, and the transfer of the user-context information from the current network to the new target network without any degradation in the QoS of the ongoing calls [27]. The handover execution also helps to facilitate the authentication and authorization of MNs to the new target network [28].

\section{MULTI-CRITERIA GROUP DECISON MAKING PROBLEM}

A group decision making (GDM) problem is defined as a decision situation, where there are two or more experts who are characterized by their own preferences/priorities trying to resolve a decision, in order to achieve a common solution [29]. A decisionmaking problem that involves several decision-makers considering several criteria is called a MCGDM. A collective group decision is reached, based of the aggregated weighting of the individual decision-maker's opinion in the group.

Consider a MCGDM problem with $N$ criteria, $M$ alternatives, $L$ group-decision members $(D M s), G=$ $\left\{D M_{1}, D M_{2}, \cdots, D M_{l}, \cdots, D M_{L}\right\}$ is the set of $L$ groupdecision members, $C=\left\{C_{1}, C_{2}, \cdots, C_{j}, \cdots, C_{N}\right\}$ is the set of criteria, and $A=\left\{A_{1}, A_{2}, \cdots, A_{i}, \cdots, A_{M}\right\}$ is the set of alternatives. The weight vectors of the criteria with respect to each group member is given by: $W^{l}=\left\{W_{1}^{l}, W_{2}^{l}, \cdots, W_{j}^{l}, \cdots, W_{N}^{l}\right\}$, $l=1,2, \cdots, L$, where each $W_{j}^{l}$ is the weight assigned to the $j^{t h}$ criterion by the $l^{\text {th }}$ group-decision member. The weight assignment satisfies $0 \leq W_{j}^{l} \leq 1$ and $\sum_{j=1}^{N} W_{j}^{l}=1$. A degree of importance described by $0 \leq \beta_{l} \leq 1$ and $\sum_{j=1}^{L} \beta_{l}=1$ for each group-decision member $D M_{l}$, is assumed. $\beta_{l}$ is the assigned weight/degree of importance of the group $D M_{l}$.

The decision matrix for the MCGDM is given by:

$$
D=\begin{gathered}
C_{1} \\
A_{1} \\
A_{2} \\
\vdots \\
A_{i} \\
\vdots \\
A_{M}
\end{gathered}\left[\begin{array}{cccccc}
x_{1,1} & x_{1,2} & \cdots & x_{1, j} & \cdots & x_{1, N} \\
& & & & & \\
x_{2,1} & x_{2,2} & \cdots & x_{2, j} & \cdots & x_{2, N} \\
\vdots & \vdots & \vdots & \vdots & \vdots & \vdots \\
x_{i, 1} & x_{i, 2} & \cdots & x_{i, j} & \cdots & x_{i, N} \\
\vdots & \vdots & \vdots & \vdots & \vdots & \vdots \\
x_{M, 1} & x_{M, 2} & \cdots & x_{M, j} & \cdots & x_{M, N}
\end{array}\right]
$$

To achieve a collective decision for the group, the group member's degree of importance and the weight vector of the criteria must be aggregated to form the aggregate-group criteria weight vector $\Psi$ for criteria of the MCGDM problem. The aggregate group criteria weight vector $\Psi$ for the $j^{\text {th }}$ criterion is:

$$
\psi_{j}=\frac{1}{L} \sum_{l=0}^{L} \beta_{l} \times W_{j}^{l}, j=1,2,3, \cdots, N .
$$

The variable, $x_{i, j}$ indicates the performance score of $i^{t h}$ alternative with respect to the $j^{\text {th }}$ criterion of the group decision matrix.
The aim of this paper is to propose a GDM methodology based on MULTIMOORA. In the next Section, the MULTMOORA technique for group calls will be discussed.

\section{MULTIMOORA TECHNIQUE}

The MULTIMOORA is a new and powerful MCDM technique that integrates three ranking approaches, namely: ratio system, reference-point system, and multiplicative form. It uses the theory of dominance to integrate the three independent ranking systems mentioned above into a single ranking output. MULTIMOORA is very simple, robust and accurate when compared with other MCDM techniques for ranking alternatives [30]. MULTIMOORA was first proposed in 2010 by Brauers, et al. [31]. In this section, the MULTIMOORA methodology is applied to solve the problem of VHO for group calls for multimode MNs in HWNs.

\subsection{Network-Selection Problem and Proposed Network-Selection Scheme}

Consider the MNs moving across HWNs that support $L$ class of calls, with $M$ available network alternatives, and $N$ network criteria. Let $A=\left\{A_{1}, A_{2}, \cdots, A_{i}, \cdots, A_{M}\right\}$ denote the set of available network alternatives, $C=\left\{C_{1}, C_{2}, \cdots, C_{j}, \cdots, C_{N}\right\}$ denote set of dynamic and static network criteria, $G=$ $\left\{D M_{1}, D M_{2}, \cdots, D M_{l}, \cdots, D M_{L}\right\}$ denote the set of supported class of group calls (decision makers) from the MNs.

Let $W^{l}=\left\{W_{1}^{l}, W_{2}^{l}, \cdots, W_{j}^{l}, \cdots, W_{N}^{l}\right\}$ be the assigned weight of importance of the network criteria by the class of call $D M_{l}$, Let $\beta=\left\{\beta_{1}, \beta_{2}, \cdots, \beta_{l}, \cdots, \beta_{L}\right\}$ be the set of importance of the group call of $L$ classes of calls.

The problem of obtaining the best network for the group calls from MNs can be solved by MULTIMOORA as follows:

- Step 1: Construct the VHO decision problem as a decision matrix $D$ defined in equation (1).

- Step 2: Obtain the aggregate group criteria weight vector, $\Psi$ from the criteria weight vector and group member degree of importance using equation (2).

- Step 3: Compute the weighted Euclidean normalized $x_{i, j}^{*}$ score for the $i^{\text {th }}$ alternative with respect to the $j^{\text {th }}$ criterion as:

$$
x_{i, j}^{*}=\frac{\psi_{j} \times x_{i, j}}{\sqrt{\sum_{i=1}^{M} x_{i, j}^{2}}} .
$$

If the larger the value of a criterion the better, such a criterion is referred to as a benefit criterion; and all benefit criteria are summed together. Likewise, if the smaller the value of the criterion the better, such a criterion is referred to as a cost criterion. And all cost criteria are summed together.

-Step 4: Compute the ratio-rank index, $y_{i}$, for the $i^{t h}$ alternative as:

$$
y_{i}=\sum_{j=1}^{k} x_{i, j}^{*}-\sum_{j=k+1}^{N} x_{i, j}^{*},
$$

where $k$ is the cardinal value of the benefit criteria and $(N-k)$ is the cardinal value of the cost criteria. The higher the value of $y_{i}$, the higher the ratio-system ranking for the $i^{\text {th }}$ alternative.

-Step 5: Compute the reference point ranking index, $y_{i}^{*}$, for the $i^{t h}$ alternative using the Min-Max metric of Tchebycheff, in as- 
cending order for the reference point system ranking,

$$
y_{i}^{*}=\min _{i}\left(\max _{j}\left(r_{j}-x_{i, j}^{*}\right)\right) .
$$

The lower the value of $y_{i}^{*}$, the higher the ranking for the $i^{t h}$ alternative in the reference point system. In the reference point system, the reference points $r_{j}=\min _{i}\left(x_{i, j}^{*}\right)$ and $r_{j}=\max _{i}\left(x_{i, j}^{*}\right)$ are defined for benefit and cost criteria, respectively.

-Step 6: Evaluates the multiplicative form ranking index $U_{i}$ for $i^{\text {th }}$ alternative as;

$$
U_{i}=\frac{A_{i}}{B_{i}}
$$

where $A_{i}=\prod_{j=1}^{k} x_{i, j}^{\psi_{j}}$ for $i^{t h}$ alternative with benefit criteria, $j=1,2,3, \cdots, k$ and $B_{i}=\prod_{j=k+1}^{N} x_{i, j}^{\psi_{j}}$ for $i^{t h}$ alternative with cost criteria, $j=k+1, k+2, k+3, \cdots, N$. The higher the multiplicative form ranking index, $U_{i}$, the higher the multiplicative form ranking of the $i^{t h}$ alternative. The MULTIMOORA ranking of the $i^{t h}$ alternative is based on its dominance in the ratio, the reference-point and the multiplication form systems.

Three types of dominance rules exist: absolute dominance, general dominance and overall dominance. The MULTIMOORA ranking technique provides a ranking score of absolute dominance, $(1,1,1)$, for the $i^{t h}$ alternative on a given ranked position, if each ranking system ranked the $i^{t h}$ alternative as 1 , for that given rank position. Given that $w<x<y<z$, the $i^{\text {th }}$ alternative with MULTMOORA score $(z, w, w)$ has general dominance over the $m^{t h}$ alternative with MULTMOORA score $(y, x, x)$. Consequently, $(w, z, w)$ is generally dominating $(x, y, x)$, and $(w, w, z)$ is generally dominating $(x, x, y)$. The $i^{\text {th }}$ alternative with $(y, y, y)$ has overall dominance over $m^{\text {th }}$ alternative with $(z, z, z)$. The ranking systems of MULTIMOORA are dimensionless. For more interesting reading on the application of dominance theory in the MULTMOORA ranking technique, readers can see [14], [32], and [33].

\subsection{Network Criteria and Degree of Importance of Group Call Specifications}

Different classes of calls require different bandwidths, security levels, and QoS from the access network available in HWNs. For example, voice calls require relatively low delay and bandwidth; while video-streaming calls require a relatively high bandwidth. These requirements are not met equally by the access networks available for all calls. Each wireless access technology has a limit on the maximum amount of mobility it can support. Hence, for MNs, the mobility is a very important factor that should be considered. Each access network will support the group calls' QoS differently at the various speeds of MNs.

In HWNs, VHO decisions for group of calls are based on static and dynamic criteria. Examples of dynamic criteria are MNs velocities, network delay, service price, network traffic load; while examples of static criteria are the security level provided for the class of calls, the maximum data rate, etc. Various networks have different dynamic performances under varying speed of the MNs. Some criteria (e.g., maximum data rate) are specified using real numbers while other criteria (e.g., battery power consumption) are specified using linguistic membership functions, as shown in Table 2 and Fig. 3 Table I and Fig. 3 show a 7-point linguistic membership function terms. The linguistic terms are defined using triangle fuzzy numbers (TFN), $a_{1}, a_{2}$ and $a_{3}$, which are the lower, middle, and upper vertex values of the TFN, respectively. The TFNs are consequently converted into their corresponding crisp numbers using the formula [34],

$$
\text { Crispvalue }=\frac{1}{6}\left(a_{1}+4 a_{2}+a_{3}\right) .
$$

Table 2.

7- Point linguistic terms

\begin{tabular}{llll}
\hline S/N & Membership function & TFN $\left(a_{1}, a_{2}, a_{3}\right)$ & Crispvalue \\
\hline 1 & Very Low (VL) & $(0,0,0.2)$ & 0.0333 \\
2 & Low (L) & $(0.1,0.2,0.3)$ & 0.2 \\
3 & Medium Low (ML) & $(0.3,0.35,0.5)$ & 0.3667 \\
4 & Medium (M) & $(0.4,0.5,0.6)$ & 0.5 \\
5 & Medium High (MH) & $(0.5,0.65,0.8)$ & 0.65 \\
6 & High (H) & $(0.7,0.8,0.9)$ & 0.8 \\
7 & Very High (VH) & $(0.8,0.9,1)$ & 0.9 \\
\hline
\end{tabular}

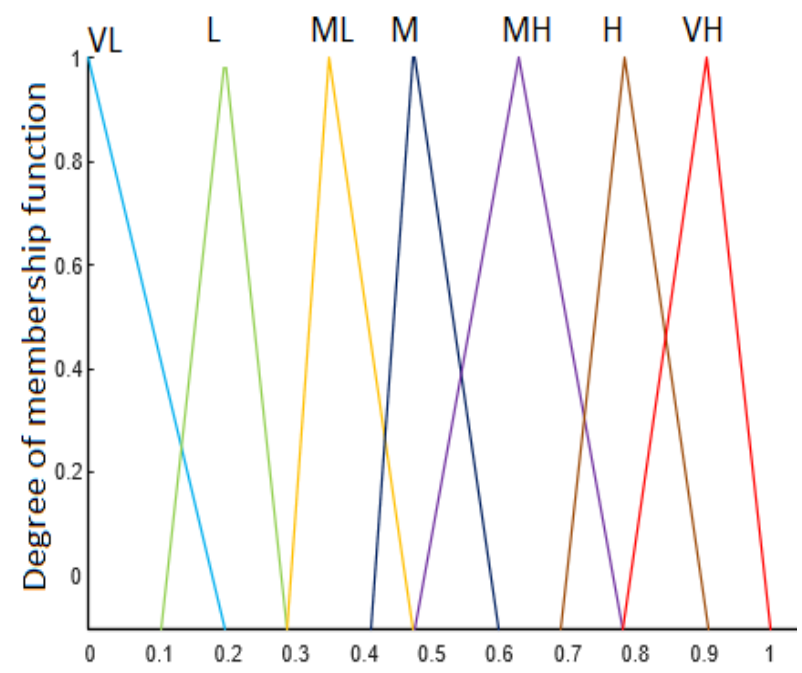

Membership functions

Fig. 3. Linguistic membership functions

In this work, the weights of classes of calls in group calls are specified in their linguistic membership terms, based on the degree of importance of the classes of calls to the users. The weights are subsequently converted into their corresponding crisp values. Different wireless network criteria have different degrees of importance for a given class of call. Different classes of calls have different weights of degree of importance in a group call to different users. The assigned weight of degree of importance for a particular wireless network criterion shows how important the criterion is to the class of call; while the assigned weight degree of important to a class of call in a group call shows how important the class of call is in the group call to the users. The weight assignments are done once by the users; and they can always be revised by the users [21]. 


\section{CASE STUDY: APPLICATION OF MULTIMOORA TO NETWORK SELECTIOM}

Considered the VHO of group calls for 1000 Multimodal MNs in HWNs that consist of WLAN $\left(A_{1}\right)$, UMTS $\left(A_{2}\right)$, and LTE $\left(A_{3}\right)$. The three integrated networks are assumed to be available for all calls. HWNs and MNs support maximum of three classes of calls in a group call. These are voice $\left(D M_{1}\right)$, file-download $\left(D M_{2}\right)$ and video-streaming $\left(D M_{3}\right)$ calls. The MNs are assumed to move across the HWNs with uniform acceleration (increasing speed from 0 to $100 \mathrm{~km} / \mathrm{h})$. The five important criteria: MN's speed $(\mathrm{km} / \mathrm{h})$ $\left(C_{1}\right)$, data rate $(\mathrm{Mbps})\left(C_{2}\right)$, security level $\left(C_{3}\right)$, network delay (ms) $\left(C_{4}\right)$, and service price $\left(C_{5}\right)$ for selecting the most feasible network for each group call are considered. Hence $L=3, M=3$, and $N=5$.

Some of the decision criteria used for VHO in heterogeneous networks are dynamic; while others are static criteria, such as MN's speed, Network service price, network load, network delays are dynamic; and they dynamically affect VHO decision for each network, however for simplification analysis of this work, the network delay is held constant.

LTE can support the highest MNs mobility among the three networks considered, while WLAN has the lowest support for MNs mobility. However, at a low pedestrian speed, such as $3 \mathrm{~km} / \mathrm{h}$, the difference in mobility support across the integrated networks might not be unduly influenced by the network with the best mobility support; whereas at a higher vehicular speed, such as $90 \mathrm{~km} / \mathrm{h}$, the choice of most suitable network for MNs will be influenced by the speed of MNs. Thus, the dynamic property of MN speed and its effect on VHOs is a crucial factor that must be taken into consideration in the design of efficient VHO for NGWNs.

Dynamic pricing has been used as an incentive to control users' behaviour and utilization of network resources [35]. Network users act autonomously and sometimes selfishly, without considering the prevailing network traffic conditions. This user's behaviour can lead to congestion in the HWNs, during rush hours or high peak periods. Network congestion can lead to loss of revenue for the network operators and dissatisfaction in user quality of experience (QoE). Network operator uses price variation (PV) to dynamically vary its network service price. This helps to control the network traffic load, network congestion and network-selection choice of MNs. Dynamic pricing can helps to achieve the dynamic loadbalancing of the HWNs. The effect of PVs, as used by the network operators to affect the relative choice of network selection cannot be ignored in the design of effective network selection algorithms for HWNs. When the network operator has no desire to control the network traffic load, the PV is de-activated/ set to zero. When an operator wishes to dynamically control the network service price, due to rising network load traffic, the PV is activated. However, network service price cannot increase infinitely with network traffic load. Network users can not be charged an infinite amount for utilizing any given network resource; hence the prices are bounded. The above network operator's service price behaviour can be modelled by a sigmoid function.

- MN's speed: The mobility criterion performance score for each network is mathematically modelled as a linear function of $\mathrm{MN}$ speed, as shown in the equations below:

$$
\begin{aligned}
\text { Speed }_{W L A N} & =V H-\frac{1}{30 k}(V H-V L) \times \text { speed }, \\
\text { Speed }_{U M T S} & =V H-\frac{1}{90 k}(V H-V L) \times \text { speed }, \\
\text { Speed }_{L T E} & =V H-\frac{1}{100 k}(V H-V L) \times \text { speed },
\end{aligned}
$$

where $k=\frac{26}{27}$.

- Dynamic price: The dynamic price criterion is mathematically modelled for each network, using the sigmoid function below:

$$
\begin{array}{r}
\text { Price }_{W L A N}=L-\frac{L-M}{1+e^{a_{W L A N}\left[P V-c_{W L A N}\right]},} \\
\text { Price }_{U M T S}=H-\frac{H-V H}{1+e^{a_{U M T S}\left[P V-c_{U M T S}\right]},} \\
\text { Price }_{L T E}=M-\frac{M-H}{1+e^{a_{L T E}\left[P V-c_{L T E}\right]},}
\end{array}
$$

where $a_{W L A N}, a_{U M T S}$ and $a_{L T E}$ are the steepness characteristics of WLAN, UMTS and LTE network dynamic price criterion, respectively. $c_{W L A N}, c_{U M T S}$ and $c_{L T E}$ are the middle-point thresholds of the dynamic price criterion for WLAN, UMTS and LTE network, respectively. The mathematical subtraction operation for two linguistic member functions and is given by:

$$
A-B=\left(a_{1}-b_{1}, a_{2}-b_{2}, a_{3}-b_{3}\right) .
$$

The benefit criteria are MN's speed, maximum data rate, and security level; while the cost criteria are network delay and network service cost. The weights of the criteria vector and degree of importance of classes of calls in the group calls used in this proposed algorithm are randomly generated to model practical wireless network operations for different users' group calls and preferences. MATLAB software is used for the simulation of the VHO of the group calls.

The decision matrix $D$, for case study is given below as:

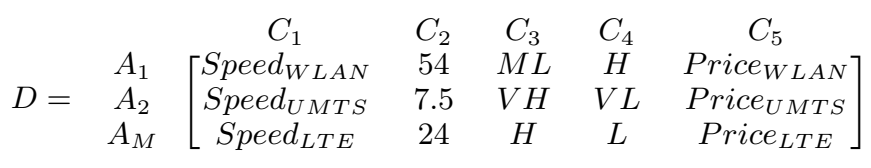

(15)

Table 3 shows the three case studies for different degrees of importance for difference classes of calls for triple calls.

Table 3.

Triple calls

\begin{tabular}{llll}
\hline & & & \\
Case study & Voice & File-download & Video-streaming \\
\hline 1 & H & L & L \\
2 & L & H & L \\
3 & L & L & H \\
\hline
\end{tabular}

In this simulation, the values of $a_{W L A N}, a_{U M T S}, a_{L T E}, c_{W L A N}$, $c_{U M T S}$ and $c_{L T E}$ are $0.29,0.102,0.25,10,90$ and 15 , respectively. 


\section{RESULTS AND DISCUSSION}

In this Section, the performance results of the proposed MULTIMOORA algorithm for triple calls using single (Fig. 4(a)-Fig.4(c)) and double (Fig.5(a)-Fig.7(c)) dynamic criteria are presented. The performance of the MULTIMOORA is validated by comparing it with TOPSIS, a well-known MCGDM algorithm. For better understanding and analysis of the results, the MN's speed is classified into three categories, namely: low MN speed $(0-30 \mathrm{~km} / \mathrm{h})$, medium MN speed (30-60 km/h) and high MN speed $(60-100 \mathrm{~km} / \mathrm{h})$. Also, the PV is classified into low PV (0-30\%), medium PV (30-60\%) and high PV $(60-100 \%)$. In the legend, MA represents MULTIMOORA.

\subsection{Single dynamic criterion: MN speed}

Fig. 4(a) presents the result of high priority voice triple calls handoff. At low speed region, for MN's speed below $10 \mathrm{~km} / \mathrm{h}$, MULTIMOORA scheme selects UMTS network as the best network for triple call, while switching over its network preference to LTE network at MN's speed above $10 \mathrm{~km} / \mathrm{h}$.

This shows that at low speed, the relative mobility performance advantage of LTE over UMTS networks is not a major decision factor for VHO decision. TOPSIS scheme prefers the LTE network all through the low speed region. TOPSIS scheme, however, fails to exploit the benefit of low network delay offered by the UMTS at MN low speed in the HWNS. At medium speed and beyond, both schemes present the LTE network as the best network alternative. However, there is a decline in preference for LTE network by the TOPSIS scheme, as the MN's speed increases above the medium speed region. This result by the TOPSIS scheme at increasing MN'speed is contradictory. It reveals the relative instability in TOPSIS; however the MULTIMOORA scheme preference for LTE network above medium speed region is constant and stable.In Fig. 4(b), MULTIMOORA and TOPSIS schemes handoff at least $50 \%$ of the high priority file-download triple calls to WLAN at any MN's speed below $23 \mathrm{~km} / \mathrm{h}$, at a low speed region, and at any MN's speed below $37 \mathrm{~km} / \mathrm{h}$ at medium speed region, respectively. At any MN's speed above $37 \mathrm{~km} / \mathrm{h}$, both schemes showed that LTE becomes clearly competitive and a better alternative than WLAN. TOPSIS shows a declining and unstable preference result for LTE networks above medium speed region. In Fig. 4(c), MULTIMOORA and TOPSIS schemes handoff atleast $50 \%$ of the high priority video-streaming triple calls to WLAN at any MN's speed below $18 \mathrm{~km} / \mathrm{h}$, at low speed region, and at any MN's speed below $36 \mathrm{~km} / \mathrm{h}$ at medium speed region, respectively. LTE network clearly outperformed WLAN and UMTS networks at above medium speed region for both schemes. However, the relative instability of TOPSIS results at high MN speed region is also noticeable.

\subsection{Double dynamic criteria: $M N$ speed and PV}

Fig. 5(a)-Fig. 5(c) present the results of high degree (priority) of importance of voice in triple calls (voice $(\mathrm{H})$, file-download $(\mathrm{L})$ and video-streaming (L) calls). In Fig. 5(a), less than $4 \%$ of the calls are admitted to WLAN through handoff at a low PV region and no group call is handoff to WLAN outside this region. In Fig. 5(b), it shows that above low PV region and for MN's speed less than $80 \mathrm{~km} / \mathrm{h}$, UMTS is selected as the best wireless network.

The results in Fig. 5(c) revealed that LTE network selections for high priority voice triple calls are highly affected by network service price dynamics. Above $20 \% \mathrm{PV}$ and at any speed less than $80 \mathrm{~km} / \mathrm{h}$, less than $55 \%$ of the triple calls are handoff to LTE. How-

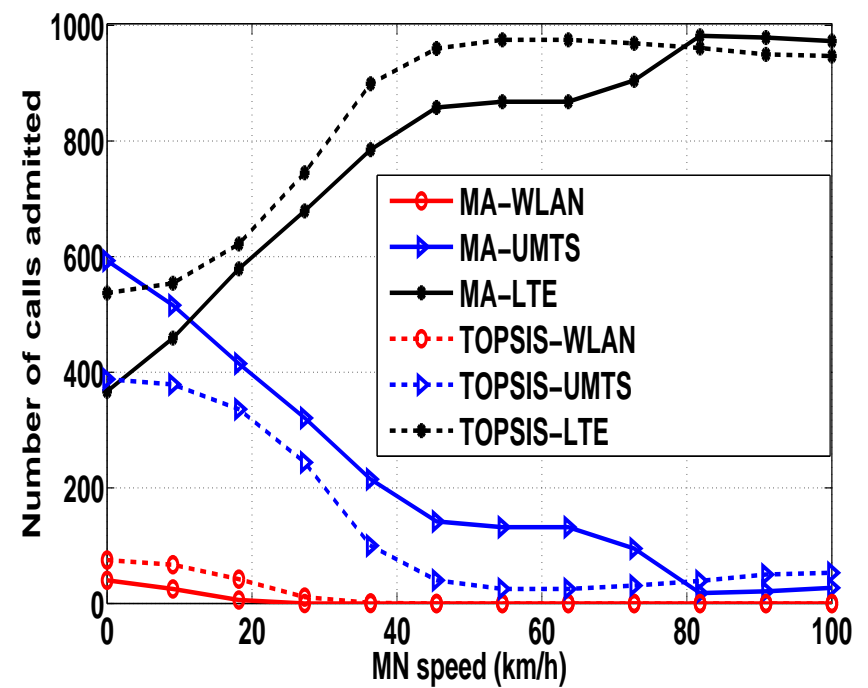

(a) High priority voice triple call handoff

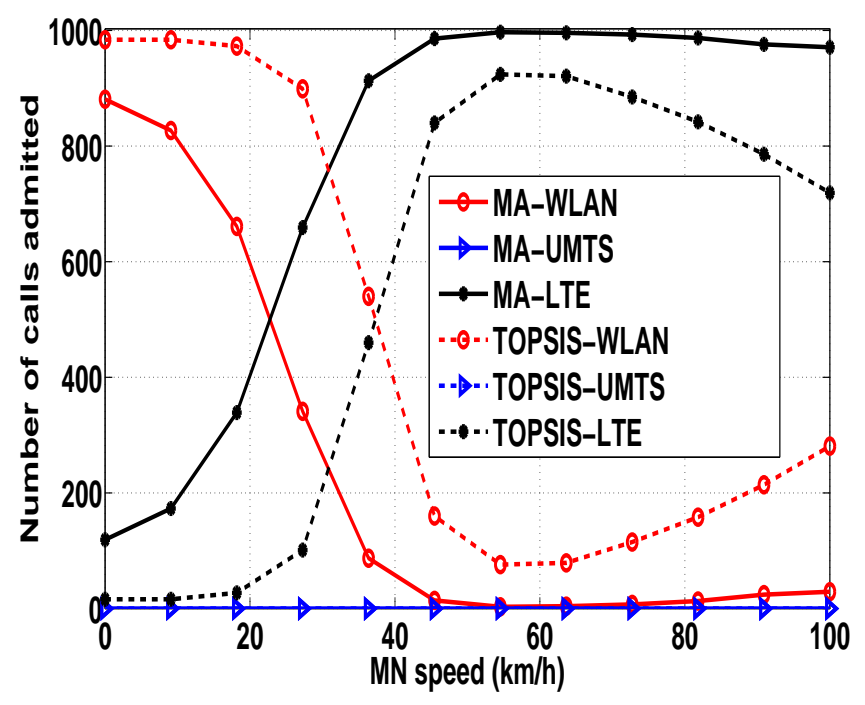

(b) High priority file-download triple call handoffs

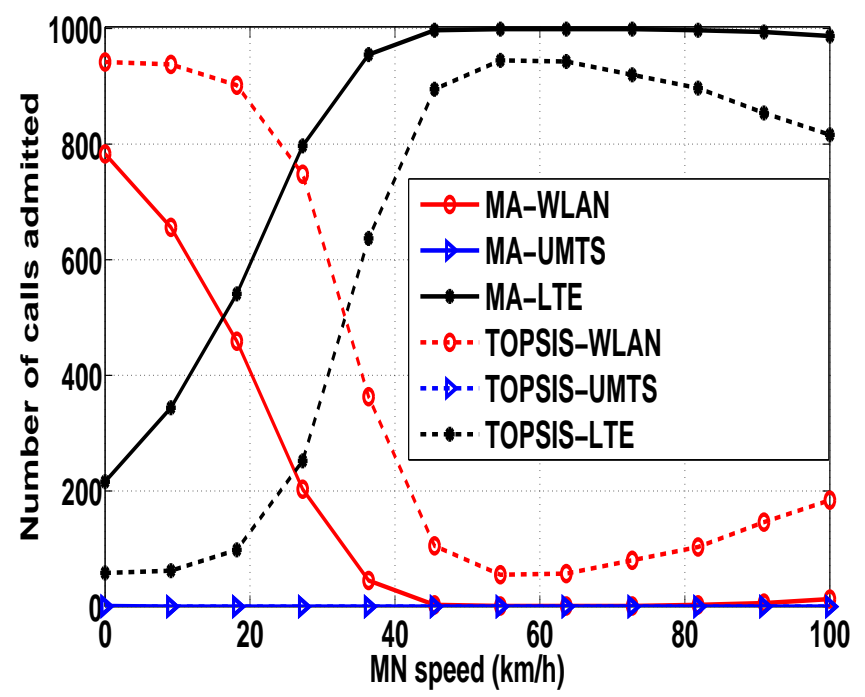

(c) High priority video-streaming triple call handoffs

Fig. 4. Triple call handoffs for high priority voice (a), file-download (b) and video-streaming (c) 


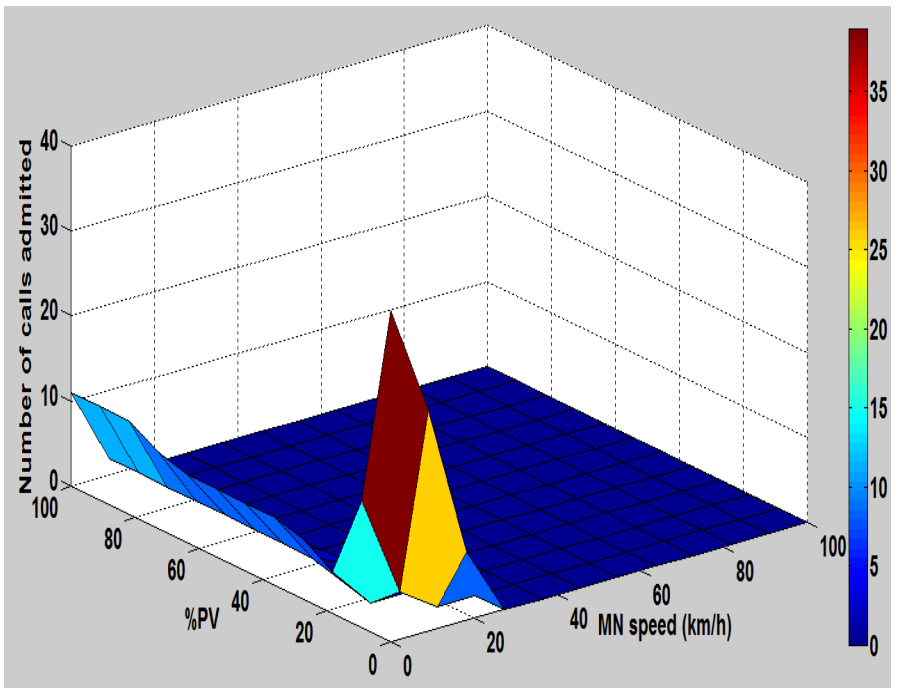

(a) WLAN :voice(H), file-download (L) and video-streaming (L)

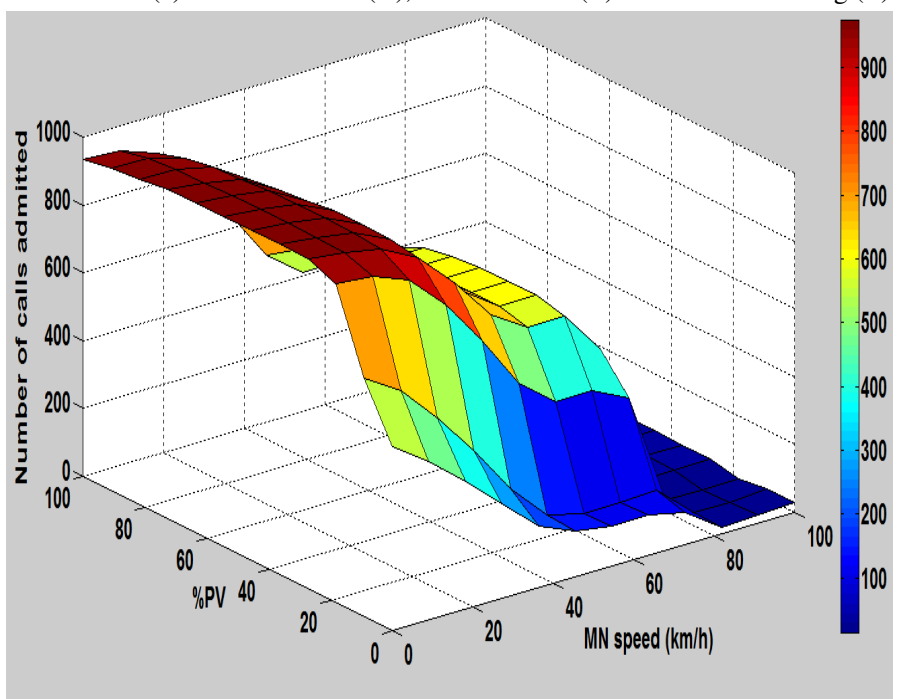

(b) UMTS :voice ( H), file-download (L) and video-streaming (L)

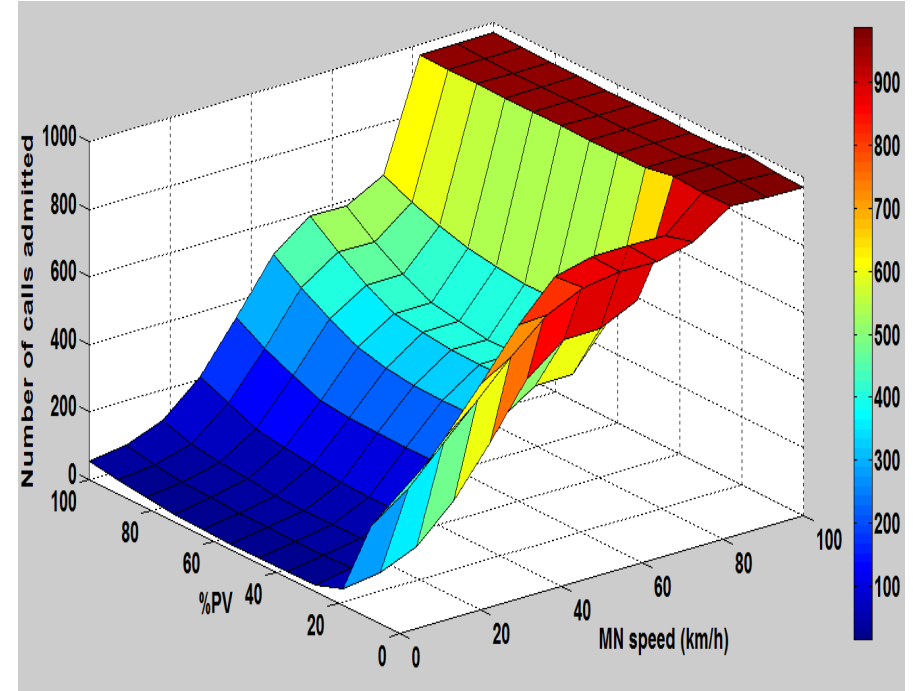

(c) LTE: voice (H), file-download (L) and video-streaming (L)

Fig. 5. High priority voice triple call handoffs to (a) WLAN), (b) UMTS and (c) LTE

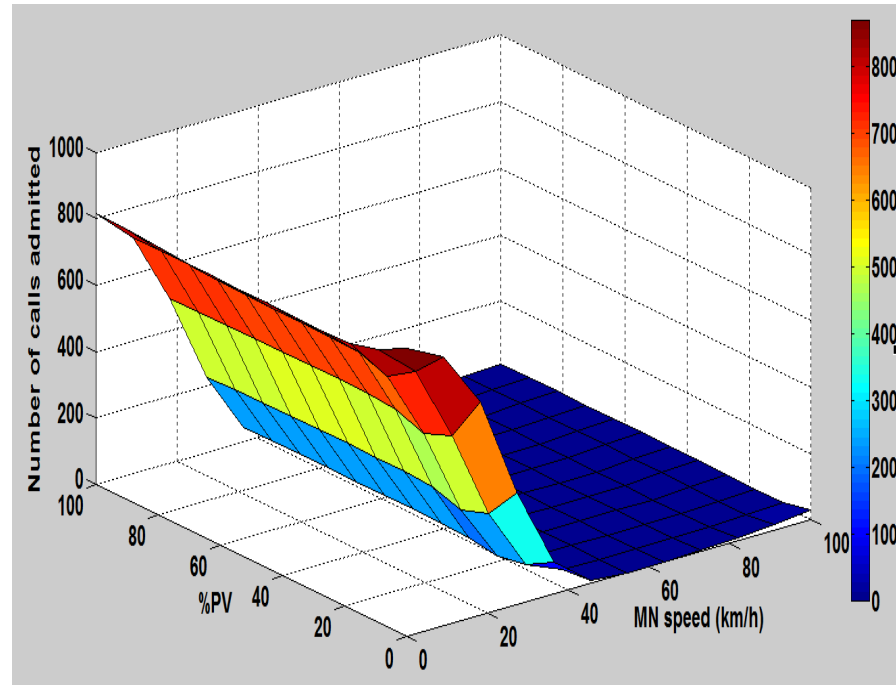

(a) WLAN :voice(L), file-download (H) and video-streaming (L)

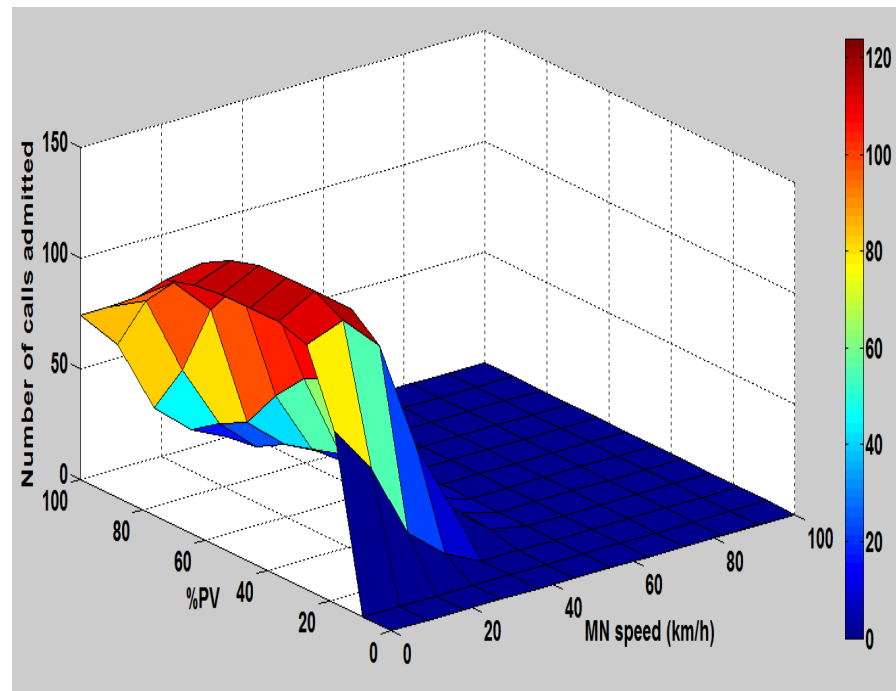

(b) UMTS :voice (L), file-download (H) and video-streaming (L)

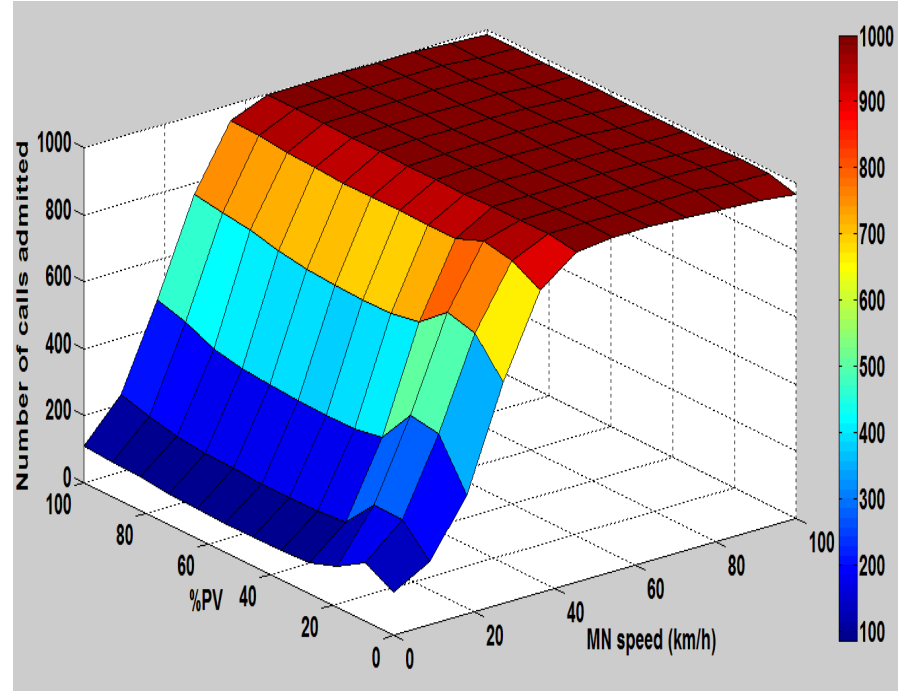

(c) LTE: voice (L), file-download (H) and video-streaming (L)

Fig. 6. High priority file-download triple call handoffs to (a) WLAN, (b) UMTS and (c) LTE 


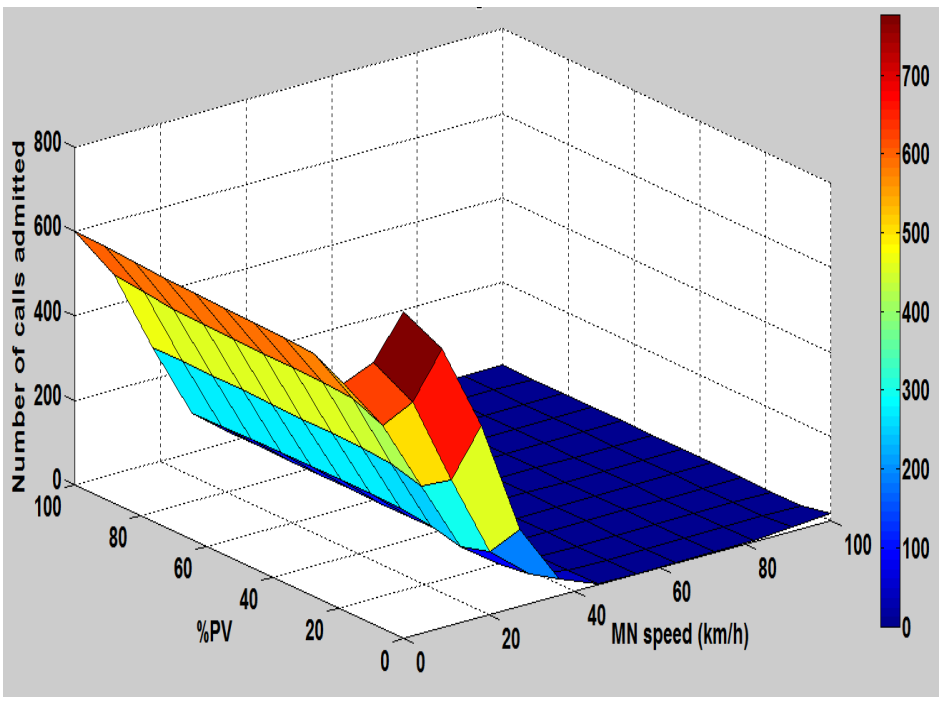

(a) WLAN :voice(L), file-download (L) and video-streaming (H)

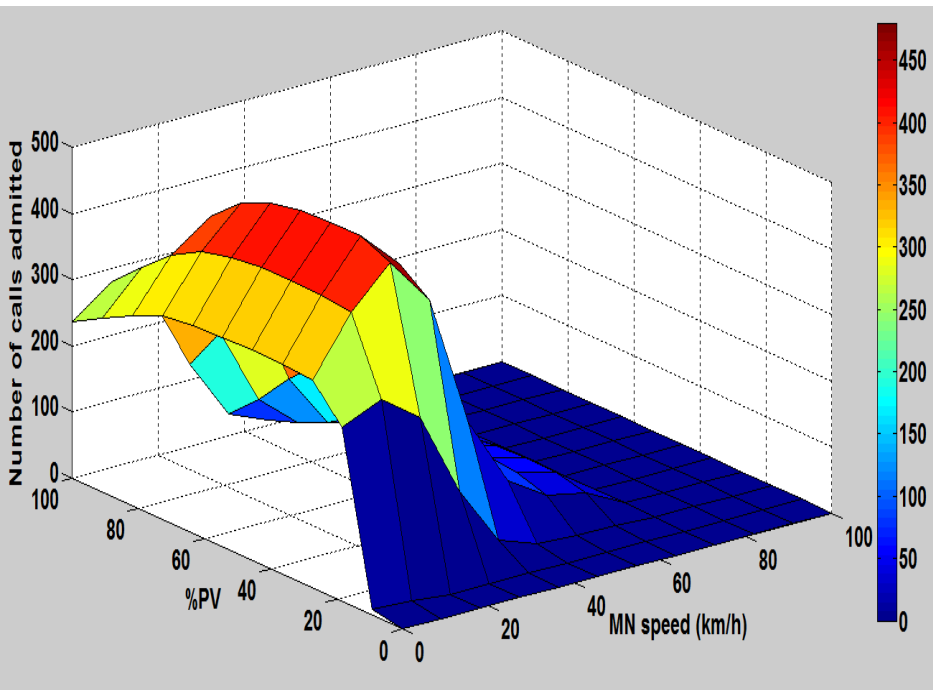

(b) UMTS : voice ( L), file-download (L) and video-streaming (H)

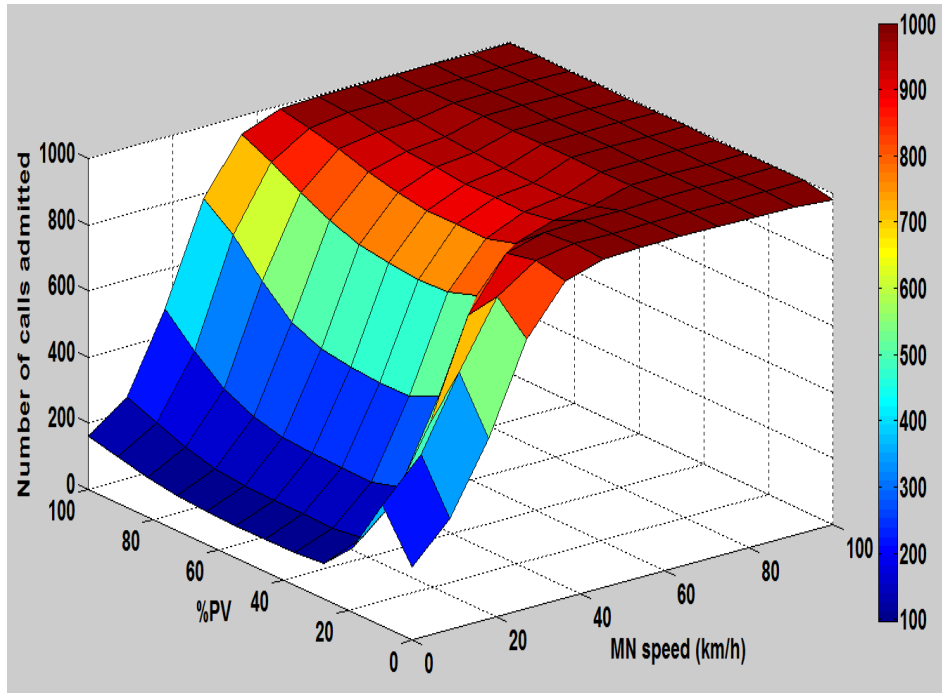

(c) LTE: voice ( L), file-download (L) and video-streaming (H)

Fig. 7. High priority video-streaming triple call handoffs to (a) WLAN, (b) UMTS and (c) LTE ever, above $60 \%$ of the triple calls are handoff to LTE any speed above $80 \mathrm{~km} / \mathrm{h}$.

Fig. 6(a)-Fig. 6(c) present the results of the high priority of filedownload in triple calls (voice $(\mathrm{L})$, file-download $(\mathrm{H})$ and videostreaming (L) calls). Fig. 6(a) reveals that at low speed region, the percentage of calls admitted to WLAN through handoff decreases from about $85 \%$ to $24 \%$. For MN speed above $40 \mathrm{~km} / \mathrm{h}$, the WLAN network is not selected for high priority file-download triple call handoff, irrespective of PV. It is shown in Fig. 6(b), that at the low speed region, the percentage of handoff calls to UMTS increases from $0 \%$ to its maximum of $14 \%$ within $40-80$ PV. However, beyond the low speed region, $0 \%$ of triple calls are handoff to UMTS. Fig. 6(c) reveals that LTE network selection for high priority filedownload triple call is marginally affected by network service price dynamics. Fig. 6(c) shows that at above $40 \mathrm{~km} / \mathrm{h}$, LTE is the best network of choice for the high degree of importance file-download triple calls compared with the rest of the networks.

Fig. 7(a)-Fig. 7(c) present the results of high priority of importance of video-streaming in triple calls (voice (L), file-download $(\mathrm{L})$ and video-streaming $(\mathrm{H})$ calls). At MN's speed above $42 \mathrm{~km} / \mathrm{h}, 0 \%$ of triple calls are handoff to the WLAN network, irrespective of any PV, as shown in Fig. 7(a). The results in Fig. 7(b) reveal that PV can be used as an incentive to raise the percentage of handoff calls to UMTS at low speed from $0 \%$ up to a maximum of $48 \%$ within PV's range of $40 \%$ to $80 \%$, at about MN's speed of $20 \mathrm{~km} / \mathrm{h}$. However, above low speed region percentage of handoff calls to UMTS drops to practically $0 \%$, irrespective of PV. The UMTS network is the most influenced by network service price dynamics. LTE network has a superlative performance for high degree of importance of video-streaming triple calls, as shown in Fig. 7(c). At MN's speed above $80 \mathrm{~km} / \mathrm{h}$, about $100 \%$ of the triple calls in the HWNs, are handoff to LTE networks, LTE network is the least influenced by network service price dynamics for high degree of importance video- streaming triple calls.

\section{CONCLUSION}

The MULTIMOORA scheme for network selection for vertical handoff of group calls has been proposed and its performance evaluated. The proposed scheme considered the dynamics of access network selection criteria in making vertical handoff decisions for group calls. The performance of MULTIMOORA is compared with TOPSIS. The of performance of TOPSIS is seen to be unstable at the high speed-region, unlike MULTIMOORA.

The effect of the dynamic criteria are investigated. The results clearly showed that the MN speed heavily influenced the selection of LTE as target network for VHO for MN's speed above medium speed region. At above medium speed region, the larger network coverage area of LTE makes it relatively very competitive network for VHO. This choice ensures lesser frequency of handover; and hence, it reduces the network overhead load. The results also showed that dynamic pricing criterion influenced the most, the selection of LTE for high priority voice triple calls and UMTS for high priority file-download and video-streaming triple calls.

The investigation into the effect of criteria interdependence on VHO of group calls by using the analytical network process (ANP) is set aside for future work,

\section{References}

[1] H. Beenish and M. Fahad, "5G a review on existing technologies," pp. 1-6, 2019. 
[2] D. Fang, Y. Qian, and R. Q. Hu, "Security for $5 \mathrm{~g}$ mobile wireless networks," 2018.

[3] L. Chen, D. Jiang, H. Song, P. Wang, R. Bao, K. Zhang, and Y. Li, "A lightweight end-side user experience data collection system for quality evaluation of multimedia communications," 2018.

[4] O. E. Falowo and H. A. Chan, "Rat selection for multiple calls in heterogeneous wireless networks using modified topsis group decision making technique," in Personal Indoor and Mobile Radio Communications (PIMRC), 2011 IEEE 22nd International Symposium on, pp. 1371-1375, 2011.

[5] A. Ajibo, F. Udechukwu, M. Chinaeke-Ogbuka, C. Nwafor, J. Nwachi-Ikpo, and C. Ani, "Review of network integration techniques for mobile broadband services in next generation network," Nigerian Journal of Technology, vol. 37, no. 2, pp. 470-479, 2018.

[6] V. K. Varma, S. Ramesh, K. D. Wong, M. Barton, G. Hayward, and J. A. Friedhoffer, "Mobility management in integrated umts/wlan networks," 2003.

[7] A. Gandhi and B. Jadhav, "Role of wireless technology for vehicular network," International Journal of Computer Science and Information Technologies (IJCSIT), vol. 3, no. 4, pp. 4823-4828, 2012.

[8] S. Datta, S. Dhar, R. N. Bera, and A. Ray, "Anp based vertical handover algorithm for vehicular communication," in Recent Advances in Information Technology (RAIT), 2012 1st International Conference on, pp. 228-234, 2012.

[9] M. Drissi and M. Oumsis, "Performance evaluation of multicriteria vertical handover for heterogeneous wireless networks," in Intelligent Systems and Computer Vision (ISCV), 2015, pp. 1-5, 2015

[10] B. Igor and S. Andrea, "Fast multiattribute network selection technique for vertical handover in heterogeneous emergency communication systems," Wireless Communications and Mobile Computing, vol. 2019, pp. 1-17, 2019.

[11] D. Maroua, O. Mohammed, and A. Driss, "Vikor for multicriteria network selection in heterogeneous wireless networks," 2016.

[12] X. Wang, B. Liu, X. Su, X. Xu, and L. Xiao, "Evolutionary game based heterogeneous wireless network selection with multiple traffics in 5g," 2019.

[13] M. Q. Khan and S. H. Andresen, "A semi and fully distributed handover algorithm for heterogeneous networks using miis," in Computers and Communications (ISCC), 2012 IEEE Symposium on, pp. 000145-000150, 2012.

[14] J. R. Gallardo-Medina, U. Pineda-Rico, and E. StevensNavarro, "Vikor method for vertical handoff decision in beyond 3g wireless networks," in Electrical Engineering, Computing Science and Automatic Control,CCE,2009 6th International Conference on, pp. 1-5, 2009.

[15] F. Bari and V. Leung, "Application of electre to network selection in a hetereogeneous wireless network environment," in Wireless Communications and Networking Conference, 2007.WCNC 2007. IEEE, pp. 3810-3815, 2007.

[16] O. Markaki, D. Charilas, and D. Nikitopoulos, "Enhancing quality of experience in next generation networks through network selection mechanisms," in Personal, Indoor and Mobile Radio Communications, 2007. PIMRC 2007. IEEE 18th International Symposium on, pp. 1-5, 2007.
[17] J. D. Martinez-Morales, U. Pineda-Rico, and E. StevensNavarro, "Performance comparison between madm algorithms for vertical handoff in $4 \mathrm{~g}$ networks," in Electrical Engineering Computing Science and Automatic Control (CCE), 2010 7th International Conference on, pp. 309-314, 2010.

[18] I. Kaliszewski and D. Podkopaev, "Simple additive weightinga metamodel for multiple criteria decision analysis methods," Expert Systems with Applications, vol. 54, pp. 155-161, 7/15 2016.

[19] P. Wang, Z. Zhu, and Y. Wang, "A novel hybrid mcdm model combining the saw, topsis and gra methods based on experimental design," Information Sciences, vol. 345, pp. 27-45, 6/1 2016

[20] O. E. Falowo and H. A. Chan, "Effect of call dynamics of a multiservice multimode terminal on rat selection in heterogeneous wireless networks," in Global Communications Conference (GLOBECOM), 2012 IEEE, pp. 5249-5253, 2012.

[21] O. E. Falowo and H. A. Chan, "Dynamic rat selection for multiple calls in heterogeneous wireless networks using group decision-making technique," Computer Networks, vol. 56, pp. 1390-1401, 3/16 2012.

[22] S. A. Sharna and M. Murshed, "Impact on vertical handoff decision algorithm by the network call admission control policy in heterogeneous wireless networks," 2012.

[23] S. Rizvi, A. Aziz, and N. M. Saad, "An overview of vertical handoff decision policies for next generation wireless networks," 2010.

[24] R. Chai, W.-G. Zhou, Q.-B. Chen, and L. Tang, "A survey on vertical handoff decision for heterogeneous wireless networks," 2009.

[25] P. Goyal, D. K. Lobiyal, and C. P. Katti, "Vertical handoff in heterogeneous wireless networks: A tutorial," 2017. ID: 1.

[26] MeriemKassar, B. Kervella, and G. Pujolle, "An overview of vertical handover decision strategies in heterogeneous wireless networks," Computer Communications, vol. 31, pp. 2607-2620, 6/25 2008.

[27] S. Mane and R. Nasri, "Handoff and network selection stratergy in next generation networks," International Journal of Advanced Research in Computer and Communication Engineering (IJARCCE), vol. 5, no. 6, pp. 304-308, 2016.

[28] R. Katru, M. Iqbal, and M. Prasad, "A survey on vertical handover in heterogeneous networks," International Journal of Innovative Research in Technology, vol. 2, no. 10, pp. 132136, 2016.

[29] Z. Yue, "Topsis-based group decision-making methodology in intuitionistic fuzzy setting," Information Sciences, vol. 277, pp. 141-153, 9/1 2014.

[30] T. Balezentis and A. Balezentis, "A survey on development and applications of the multi-criteria decision making method multimoora," Journal of Multi-Criteria Decision Analysis, vol. 21, no. 3-4, pp. 209-222, 2014.

[31] W. Brauers and E. K. Zavadskas, "Project management by multimoora as an instrument for transition economies," Technological and economic development of economy, Baltic Journal on Sustainability, vol. 16, no. 1, pp. 5-24, 2010.

[32] W. K. M. Brauers and EdmundasKazimierasZavadskas, "Multimoora optimization used to decide on a bank loan to buy property," Technological and Economic Development of Economy, vol. 17, pp. 174-188, 03/22; 2015/06 2011. doi: 10.3846/13928619.2011.560632; 09 . 
[33] W. K. M. Brauers, E. K. Zavadskas, and SimonaKildien, "Was the construction sector in 20 european countries anti-cyclical during the recession years 2008-2009 as measured by multicriteria analysis (multimoora)?," Procedia Computer Science, vol. 31, no. 0, pp. 949-956, 2014.

[34] T. Kaya and CengizKahraman, "Multicriteria decision making in energy planning using a modified fuzzy topsis methodology," Expert Systems with Applications, vol. 38, pp. 65776585, 62011.

[35] O. E. Falowo, SheraliZeadally, and H. A. Chan, "Dynamic pricing for load-balancing in user-centric joint call admission control of next-generation wireless networks," International Journal of Communication Systems, vol. 23, no. 3, pp. 335$368,2010$. 